\title{
ABiMed: Towards an Innovative Clinical Decision Support System for Medication Reviews and Polypharmacy Management
}

Abdelmalek MOUAZER ${ }^{\mathrm{a}}$, Romain LEGUILLON ${ }^{\mathrm{a}}$, Bertille LEROY ${ }^{\mathrm{d}}$, Karima SEDKI ${ }^{\mathrm{a}}$, Christian SIMON ${ }^{\mathrm{b}}$, Hector FALCOFF ${ }^{\mathrm{c}}$, Matthieu SCHUERS d,a, Sophie DUBOIS ${ }^{\mathrm{e}}$, Cédric BOUSQUET ${ }^{\mathrm{a}}$, Lina SOUALMIA ${ }^{\mathrm{f}, \mathrm{a}}$, Catherine LETORD $^{\mathrm{a}}$, Stéfan DARMONI ${ }^{\mathrm{a}, \mathrm{g}}$, Jacques BOUAUD ${ }^{\mathrm{a}}$, Brigitte SÉROUSSI ${ }^{\mathrm{a}}$, Rosy TSOPRA ${ }^{\mathrm{h}, \mathrm{i}}$ and Jean-Baptiste LAMY ${ }^{\mathrm{a}, 1}$

a Laboratoire d'Informatique Médicale et d'Ingénierie des Connaissances en e-SantéLIMICS, Université Sorbonne Paris Nord, Sorbonne Université, INSERM UMR 1142, F-93000, Bobigny, France

' EIG Santé, 49070 Beaucouzé, France

' Société de Formation Thérapeutique du Généraliste (SFTG), Paris, France

${ }^{\mathrm{d}}$ Département de Médecine Générale, Université de Rouen, France

${ }^{\mathrm{e}}$ CPTS Paris 13, 75013 Paris, France

${ }^{\mathrm{f}}$ Normandie Univ, UNIROUEN, LITIS EA 4108, F-76000 Rouen, France

${ }^{\mathrm{g}}$ Department of Biomedical Informatics, Rouen University Hospital, France

${ }^{\mathrm{h}}$ INSERM, Université de Paris, Sorbonne Université, Centre de Recherche des

Cordeliers, Information Sciences to support Personalized Medicine, F-75006 Paris, France

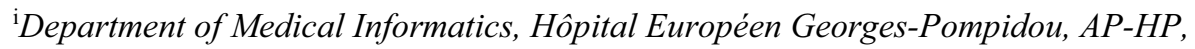
Paris, France

\begin{abstract}
Polypharmacy in elderly is a public health problem with both clinical (increase of adverse drug events) and economic issues. One solution is medication review, a structured assessment of patients' drug orders by the pharmacist for optimizing the therapy. However, this task is tedious, cognitively complex and error-prone, and only a few clinical decision support systems have been proposed for supporting it. Existing systems are either rule-based systems implementing guidelines, or documentary systems presenting drug knowledge. In this paper, we present the ABiMed research project, and, through literature reviews and brainstorming, we identified five candidate innovations for a decision support system for medication review: patient data transfer from GP to pharmacists, use of semantic technologies, association of rule-based and documentary approaches, use of machine learning, and a two-way discussion between pharmacist and GP after the medication review.
\end{abstract}

Keywords. Polypharmacy, Medication review, Clinical decision support system

\footnotetext{
${ }^{1}$ Corresponding Author, Jean-Baptiste LAMY; E-mail: jean-baptiste.lamy@univ-paris13.fr.
} 


\section{Introduction}

Many elderly patients suffer from multiple chronic disorders and receive polypharmacy (i.e. 5 drugs or more). It is both a public health and an economic issue. Each new drug administered in polypharmacy increases the risk of adverse events by $12-18 \%$ [1], e.g. recently, many drugs (including anti-inflammatory) increased the symptoms of Covid19. Both the drugs and the hospitalization caused by the adverse events have a high cost for health insurances. Finally, reducing polypharmacy is also beneficial for achieving the UNO goal of a more sustainable consumption.

One solution is medication reviews (MR) [2], a structured assessment of patients' drug orders for optimizing the drug use and to improve health outcomes. In MR, the pharmacist assesses the patient observance and tolerance, and wrote a synthesis with recommendations for the GP. MR can reduce polypharmacy and save money, without lowering the quality of care [3]. However, few pharmacists are engaged in MR, because it is a tedious and highly cognitive task that requires specific knowledge in geriatrics. It requires to collect the patient data (often available in the GP's patient records, but not in the pharmacist's ones), to assess the interactions and adverse effects of 5-20 drugs, to identify inappropriate or missing drugs (e.g. by manually applying the numerous rules in the existing recommendations [4]) and to write the synthesis.

Few clinical decision support systems (CDSSs) were proposed for MR. Rule-based systems aim at detecting inadequate prescriptions, either by implementing guidelines [5] or by letting users build their rule base [6]. Documentary systems provide information on demand. They typically present the drugs the patient takes on a table, and displays their interactions and potential adverse effects, e.g. [7]. Semantic web technologies are known to improve CDSSs [8], but have not yet been applied to MR.

ABiMed (Aide au Bilan de Médication, medication review support in English) is a research project funded by the French research agency. The objectives of ABiMed are to design and evaluate a clinical decision support system for facilitating medication reviews and reducing polypharmacy, which combines rule-based and documentary approaches. The system is destined to pharmacists (for MR) and GPs (for preventing polypharmacy when prescribing). In this paper, we present the project and the five candidate innovations we identified for decision support for medication reviews.

\section{Methods}

Candidate innovations for a CDSS devoted to MR and polypharmacy were identified through literature reviews and multidisciplinary collective brainstorming sessions. Two systematic literature reviews were performed, the first targeting guidelines and recommendations for MR and the second targeting CDSSs for MR. These reviews are still under progress before publication.

Brainstorming sessions involved all partners of the project: an academic research lab in medical informatics (LIMICS), an enterprise developing medical software (EIG Santé), and two clinical partners: an association performing continuing medical education (SFTG) and a territorial professional health community (CPTS 13). Participant backgrounds include pharmacy, primary care, hospital medicine, and computer science; with several persons having a double competence. Due to Covid-19, meetings were performed online. 


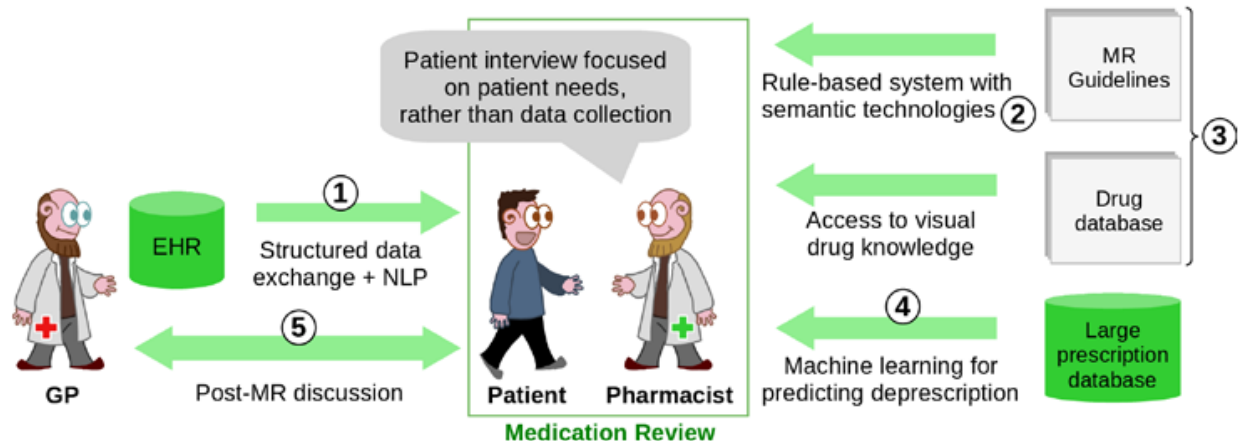

Figure 1. General overview of ABiMed (numbers match those in the text).

\section{Results}

We identified five candidate innovations that we will include in our CDSS (Figure 1):

(1) We will automatically extract patient data from GP electronic health records (EHR) and transfer it to the pharmacist. Current CDSSs are not linked to EHR when they target pharmacists, due to the wide number of EHR vendors. We will rely on the French shared health records (DMP) for accessing data, and on natural language processing (NLP) tools [9] for extracting concepts from plain text medical reports.

(2) We will use semantic technologies, such as formal ontologies and the SPARQL query language. They facilitate the management of several granularity levels, e.g. specific disorders in patient data $v s$ more general disorder categories in rules from guidelines.

(3) We will combine a rule-based system implementing the recommendations from guidelines for MR with a documentary system presenting drug knowledge. It will rely on the visual analytics we developed previously for clinical trial data [10]. Preliminary results on the visualization of drug-drug interactions with graphs are promising [11].

(4) We will also rely on machine learning for learning deprescribing behaviors from large prescription databases such as the reimbursement data of the French health insurance. The objective is to learn real-life deprescription rules, possibly different from those in the guidelines. For instance, guidelines recommend stopping benzodiazepines, but GPs said that patients often ask for them at the next consultation. Thus, we may learn that benzodiazepines are not the best candidates for deprescription.

(5) We will experiment a two-way discussion between the pharmacist and the GP after the MR, instead of the traditional one-way communication mode, through the textual synthesis sent by the pharmacist to the GP.

\section{Discussion and conclusion}

Through literature reviews and brainstorming, we identified five innovations that have the potential of improving decision support for MR and polypharmacy management. There is a mix of technical innovation (e.g. 4), combination of existing technics (3) or application of known technics to MR $(1,2)$, and organizational innovation (5). These innovations still have to be properly implemented and evaluated in clinical settings. The 
ABiMed project includes both in vitro and in vivo evaluation, aimed at showing a positive impact of the proposed CDSS on medical prescriptions.

In the literature, EHR data extraction was proposed for CDSSs for physicians, but rarely for pharmacists, because the data is not at the pharmacist office. Semantic technologies are commonly used for CDSSs, but they have rarely been implemented for MR. A few systems tried to associate rule-based and documentary approaches, such as PRIMA-eDS [12]. Finally, no machine learning approaches have been proposed for MR and very few for drug therapy in general, despite a recent study showed that machine learning was better than traditional methods (i.e. if-then rules implemented from guidelines) for the automatic detection of at-risk prescriptions at hospital [13].

\section{Acknowledgments}

This work was funded by the French Research Agency (ANR) through the ABiMed project [grant number ANR-20-CE19-0017-02].

\section{References}

[1] Calderón-Larrañaga A, Poblador-Plou B, González-Rubio F, et al. Multimorbidity, polypharmacy, referrals, and adverse drug events: are we doing things well? The British journal of general practice: the journal of the Royal College of General Practitioners. 2012;62(605):e821-6.

[2] Silva RDOS, Macêdo LA, Santos GAD, Aguiar PM, de Lyra DP. Pharmacist-participated medication review in different practice settings: Service or intervention? An overview of systematic reviews. PloS one. 2019;14(1):e0210312.

[3] Zermansky A G, Petty D R, Raynor D K, Freemantle N, Vail A, Lowe C J. Randomised controlled trial of clinical medication review by a pharmacist of elderly patients receiving repeat prescriptions in general practice. BMJ (Clinical research ed). 2001;323(7325):1340-3.

[4] Roux B, Berthou-Contreras J, Beuscart JB, Charenton-Blavignac M, Doucet J, Fournier JP, et al. REview of potentially inappropriate MEDIcation pr[e]scribing in Seniors (REMEDI[e]S): French implicit and explicit criteria. Eur J Clin Pharmacol. 2021.

[5] Meulendijk MC, Spruit MR, Drenth-van Maanen AC, Numans ME, Brinkkemper S, Jansen PAF, et al. Computerized Decision Support Improves Medication Review Effectiveness: An Experiment Evaluating the STRIP Assistant's Usability. Drugs \& aging. 2015;32(6):495-503.

[6] Bindoff I, Stafford A, Peterson G, Kang B H, Tenni P. The potential for intelligent decision support systems to improve the quality and consistency of medication reviews. J Clin Pharm Ther. 2012;37(4):452-8

[7] Koskela T, Sandström S, Mäkinen J, Liira H. User perspectives on an electronic decision-support tool performing comprehensive medication reviews - a focus group study with physicians and nurses. BMC medical informatics and decision making. 2016;16:6.

[8] Dissanayake PI, Colicchio TK, Cimino JJ. Using clinical reasoning ontologies to make smarter clinical decision support systems: a systematic review and data synthesis. J Am Med Inform Assoc. 2019.

[9] Siefridt C, Grosjean J, Lefebvre T, Rollin L, Darmoni S, Schuers M. Evaluation of automatic annotation by a multi-terminological concepts extractor within a corpus of data from family medicine consultations. Int J Med Inf. 2020;133:104009.

[10] Lamy JB. A data science approach to drug safety: Semantic and visual mining of adverse drug events from clinical trials of pain treatments. Artif Intell Med. 2021;115:102074.

[11] Mouazer A, Sedki K, Tsopra R, Lamy JB. Visualization of drug interactions for supporting medication review. In: Studies in health technology and informatics (ICIMTH). vol. 272; 2020. p. 107-10.

[12] Rieckert A, Sommerauer C, Krumeich A, Sönnichsen A. Reduction of inappropriate medication in older populations by electronic decision support (the PRIMA-eDS study): a qualitative study of practical implementation in primary care. BMC family practice. $2018 ; 19(1): 110$.

[13] Corny J, Rajkumar A, Martin O, Dode X, Lajonchère JP, Billuart O, et al. A machine learning-based clinical decision support system to identify prescriptions with a high risk of medication error. J Am Med Inform Assoc. 2020;27(11):1688-94. 